\title{
Etude hydrosédimentaire des plages de Morgat
}

\author{
Nicholas Grunnet \\ DHI Water \& Environment \\ Agern Allé 5, DK-2970 Hørsholm, Danemark \\ Christophe Chevalier \\ DHI Eau et Environnement \\ 1 rue Du Guesclin, 44000 NANTES
}

\begin{abstract}
Résumé
L'objet de cette communication est la modélisation numérique de la dynamique sédimentaire des plages de Morgat dans le Finistère en Bretagne. Suite à l'extension du port de Morgat avec la construction d'une grande digue au large, l'équilibre sédimentaire des plages avoisinantes s'est trouvé perturbé. Une reproduction de l'évolution de la plage de Morgat est obtenue avec le logiciel MIKE 21 de DHI. La modélisation comprend les études de propagation de la houle, de courantologie et de transport sédimentaire avec prise en compte de la variation du plan d'eau (marnage de l'ordre de $4 \mathrm{~m}$ ) et des aspects bidimensionnels du transport littoral. Le calage du modèle numérique s'appuie à la fois sur les périodes antérieure et postérieure à la construction de la digue extérieure. Un aménagement du site est proposé pour restaurer et assurer la stabilité des plages de Morgat.
\end{abstract}

\begin{abstract}
This paper deals with the numerical modelling of sediment transport of Morgat beach, Brittany, France. Following the extension of Morgat harbour with a large breakwater, the equilibrium of the beach became severely disturbed. A good representation of the beach evolution was achieved by numerical modelling using MIKE 21 software developed by DHI. The investigations include modelling of nearshore wave transformation, hydrodynamic flow and sediment transport. The 2D model was calibrated for the period before and after the extension of the harbour and included water level variations (with tidal range on the order of $4 \mathrm{~m}$ ). A solution is proposed to restore and stabilise the beach.
\end{abstract}

\section{Introduction}

L'anse de Morgat est située sur le littoral Sud de la presqu'île de Crozon. Cette anse se trouve inscrite dans un site naturellement abrité des houles océaniques au niveau de l'extrémité Nord de la Baie de Douarnenez. L'anse de Morgat s'étend sur un peu plus de $1500 \mathrm{~m}$ et se trouve encadrée par deux pointes rocheuses (Figure 1). Dans le fond de l'anse se situe un ensemble de 2 plages sableuses séparées par la pointe de Rulianec : la plage de Morgat au Sud-ouest et la plage de Portzic au Nord-est. Le port de Morgat occupe l'extrémité Sud de l'anse ; la digue du large, construite au début des années 70, parachève la protection des bassins portuaires du port de plaisance contre l'agitation.

Avant l'extension du port de Morgat réalisé en 1971/72, l'ensemble des plages de l'anse de Morgat était en équilibre dynamique avec une plage de largeur uniforme dans le fond de l'anse. La construction de la grande digue du large va bousculer la 
dynamique sédimentaire des plages du fond de l'anse: la partie centrale et orientale de cet ensemble s'est alors considérablement érodée (érosion de l'ordre de $350000 \mathrm{~m}^{3}$ en 25 ans, [1]), cependant qu'à l'opposé le secteur occidental s'engraissait.
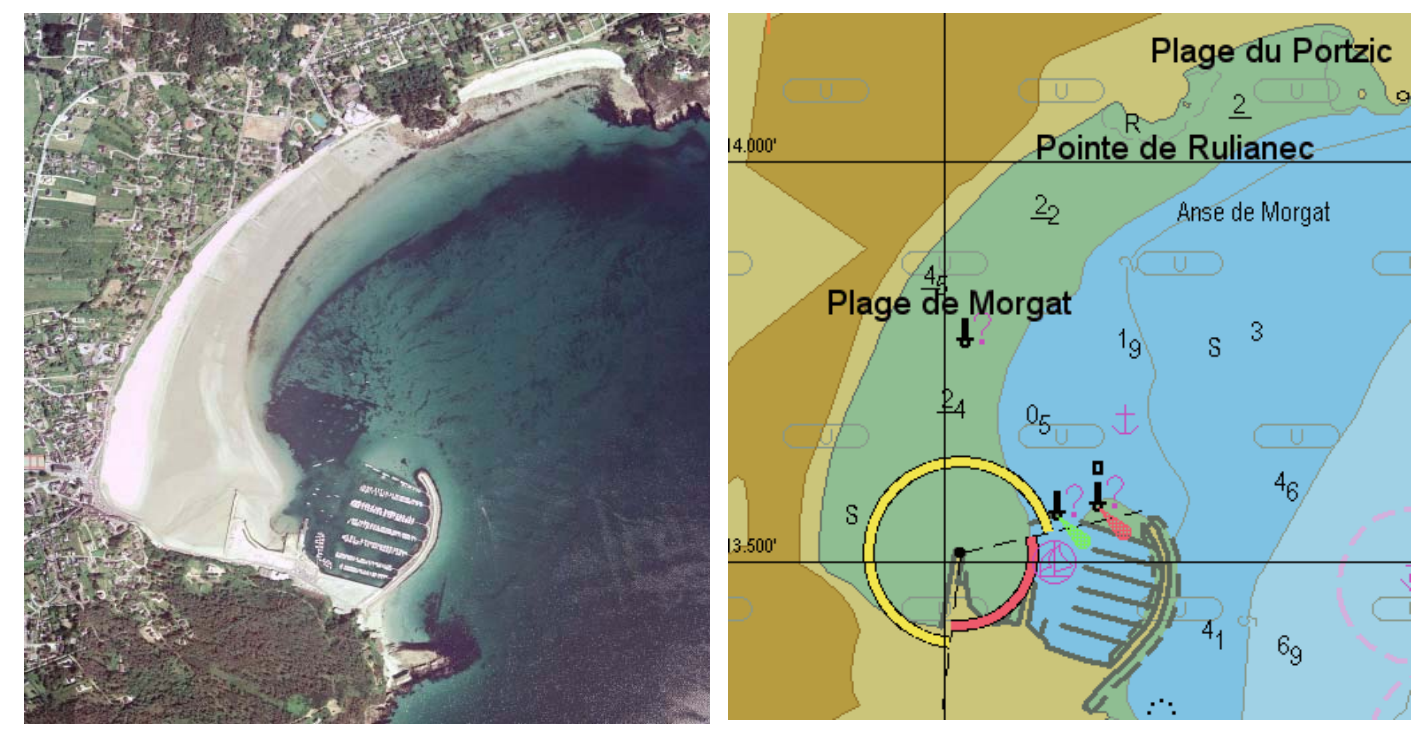

Figure 1: Vue aérienne de l'anse de Morgat - situation actuelle (à gauche) et plages du fond de l'anse (à droite).

Dans la cadre du projet de réaménagement global du site, DHI a mené une étude hydro sédimentaire détaillée du secteur de Morgat afin de déterminer le meilleur scénario d'aménagement. Cette étude s'appuis sur une modélisation numérique 2D de la dynamique sédimentaire prenant en compte la houle, les courants engendrés par son déferlement, les variations de la hauteur du plan d'eau. Les principales étapes de cette modélisation sont présentées à suivre: choix de conditions de houle représentatives, construction et calage des différents modèles (houle, courant et transport sédimentaire). Le modèle a permis ainsi de mettre en évidence les mécanismes de la rupture de l'équilibre sédimentaire et de déterminer un scénario d'aménagement permettant d'obtenir un nouvel état d'équilibre sédimentaire de l'anse de Morgat.

\section{Caractéristiques environnementales}

Les sables sont largement compris entre 0.1 et $0.3 \mathrm{~mm}$ et ont un diamètre moyen de $0.2 \mathrm{~mm}$. Dans les petits fonds jusqu'a $-5 \mathrm{~m}$ C.M., les sables deviennent fins à très fins de l'ordre de 0.12 à $0.14 \mathrm{~mm}$.

Dans l'anse de Morgat, le niveau de mi-marée moyenne (coefficient 70) se situe à +3.77 m C.M., le marnage correspondant étant de $4.25 \mathrm{~m}$. Les courants de marée y restent très modérés, même en vive-eau moyenne, ceux-ci restent bien inférieurs au demi-nœud, [2]. Les courants de marée n'ont pas fait l'objet d'une analyse car leur influence sur le transport sédimentaire à la côte est secondaire par rapport à l'influence de la houle et le clapot.

Aucune donnée sur les états de mer dans l'anse de Morgat n'a pu être trouvée. 
Deux modèles numériques de propagation ont ainsi été mis en œuvre au préalable afin d'obtenir une bonne description de la houle sur le site d'étude à partir de la houle du large: un modèle global comprenant une partie de la mer d'Iroise et pour la Baie de Douarnenez et un autre plus local pour l'anse de Morgat. Les simulations de la propagation de la houle du large et du clapot généré localement ne sont pas présentées dans cette communication.

\section{Détermination préalable de conditions représentatives de forçage}

Afin de limiter le nombre de simulations 2D très coûteuses en temps de calculs, une modélisation simplifiée du transit littoral est d'abord mise en œuvre afin de déterminer les conditions les plus représentatives du transport sédimentaire. Le choix des conditions représentatives est basé sur des calculs de transit littoral de sorte que le transport net le long de la côte résultant de la climatologie entière soit comparable - en intensité et en direction - au transport déduit des seules conditions représentatives. Le transit littoral a été calculé avec le modèle mathématique LITPACK basé sur l'hypothèse de conditions quasi-stationnaires le long de la côte. Ce module permet de calculer la transformation de la houle à travers la zone de déferlement, le courant de houle et le transport littoral résultant. Les données d'entrée du modèle sont les statistiques de houle proche de la côte, des mesures de profils de plage et de granulométrie. Le transit littoral est le résultat des contributions unitaires de toutes les conditions de houle (hauteur, période et direction), conditions pondérées par leur fréquence d'occurrence.

La schématisation des conditions de houle se base sur les caractéristiques de houle dans la zone non abritée par la digue du large dans le Nord-est de l'anse, face à la plage de Portzic. Le résultat des simulations LITPACK est présenté graphiquement sur la Figure 2.
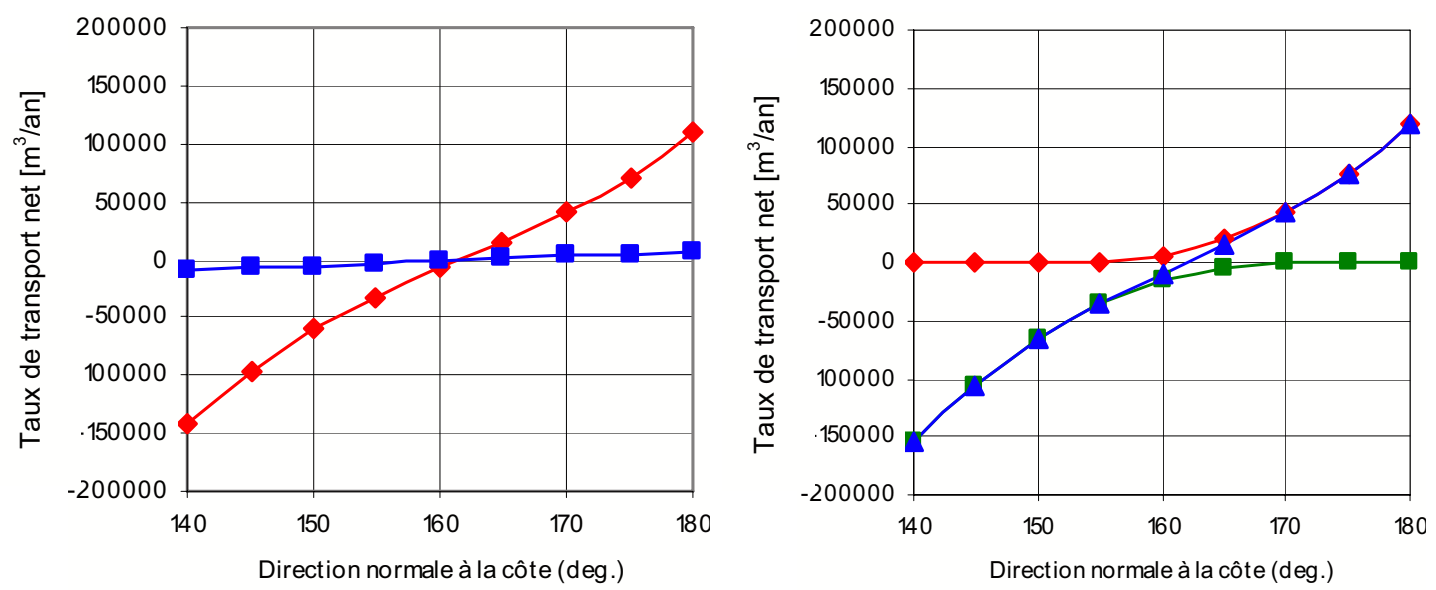

Figure 2: A gauche : taux de transport littoral net résultant de la houle (rouge) et du clapot (bleu) en fonction de la direction normale à la côte. A droite : taux de transport vers l'Ouest (rouge) et l'Est (vert) et transport total (bleu) en fonction de la direction normale à la côte.

L'orientation d'équilibre de la plage de Portzic, i.e. l'orientation correspondant à un transport net nul, est environ $162^{\circ}$. Ceci correspond effectivement à l'orientation moyenne des contours topographiques et bathymétriques de la plage 
de Portzic actuelle (voir également la figure 1). Il est intéressant de noter que la plage de Portzic avant la construction de la digue du large, soit pour un équilibre antérieur de la dynamique sédimentaire de l'anse, avait la même orientation. Ainsi, a l'opposé de la plage de Morgat qui a progressivement basculée, la plage de Portzic a gardé son orientation d'équilibre malgré l'érosion qu'elle a subit. L'absence de changement d'orientation de la plage de Portzic est par ailleurs confirmée par l'étude de modélisation de propagation de houle qui démontre que le port de plaisance n'a pas engendré de changement dans les conditions de forçage face à cette plage.

Deux observations principales découlent de l'analyse de ces résultats: la dominance de la houle (Figure 2 - Gauche) et le forçage très étroit (non présenté ici). La dominance que la houle exerce sur la mobilisation des sédiments permet de conclure qu'aucun composant du clapot n'est véritablement représentatif du transit littoral. Ainsi les conditions représentatives ont été choisies uniquement parmi les composants de houle et uniquement pour une direction d'incidence le long de l'isobathe $-10 \mathrm{~m}$ CM: deux conditions représentatives sont jugées suffisantes pour décrire la capacité de transport de la climatologie entière (voir tableau 1). Le choix de la hauteur significative de chacune de ces conditions est basé sur la rose du transport littoral.

Tableau 1: Conditions représentatives de la climatologie entière à l'isobathe $-10 \mathrm{~m}$ CM face à

\begin{tabular}{|c|c|c|c|c|}
\hline Condition & $\mathrm{H}_{\mathrm{s}}[\mathrm{m}]$ & $\mathrm{T}_{\mathrm{p}}[\mathrm{s}]$ & MWD [deg] & Fréq. [\%] \\
\hline 1 & 1.02 & 13.2 & 190 & 9.6 \\
\hline 2 & 1.69 & 14.5 & 190 & 1.4 \\
\hline
\end{tabular}

\section{Présentation de la modélisation hydrosédimentaire}

Une étude bidimensionnelle de la propagation de la houle, de la courantologie et du transport sédimentaire résultant est mise en œuvre ; celle-ci est effectuée avec les modules PMS, HD et ST de la suite logicielle MIKE 21.

Le module de propagation de la houle MIKE 21 PMS est mis en œuvre pour la modélisation de la propagation de la houle de l'entrée de l'anse de Morgat vers la ligne de rivage. Compte tenu de la complexité du trait de côte encadré entre pointes rocheuses et aménagements portuaires, il est impératif de prendre en compte les phénomènes de réfraction et de diffraction de la houle dans cette anse. MIKE 21 PMS est un modèle de réfraction-diffraction basé sur l'approximation parabolique de l'équation de faible pente. Le modèle prend en compte les phénomènes de réfraction, de shoaling, de diffraction perpendiculaire à la direction prépondérante de la houle et de dissipation d'énergie dû au déferlement et au frottement de fond. Le modèle prend également en compte les phénomènes de houle aléatoire et d'étalement directionnel. Un spectre d'énergie JONSWAP correspondant aux conditions présentées dans le Tableau 1 est imposé sur la frontière offshore. Les simulations de houle sont réalisées pour un cycle de marée moyenne et sont menées toutes les heures dans le but de prendre en compte l'évolution du niveau d'eau. 
Le module HD de la suite MIKE 21 est mis en œuvre pour la modélisation de la courantologie dans l'anse de Morgat. MIKE 21 HD est un modèle bidimensionnel pour les calculs hydrodynamiques qui permet de simuler les variations du niveau de la surface libre et du courant moyenné sur la colonne d'eau, en fonction des paramètres de forçage des zones côtières et océaniques. Ce module est utilisé principalement pour simuler de nombreux phénomènes hydrauliques dont la marée, les courants induits par le vent et la houle ainsi que les surcotes. Pour les conditions du Tableau 1, les courants sont calculés à partir des contraintes de radiation issues du modèle PMS. Le niveau d'eau aux frontières latérales est modifié localement afin de prendre en compte la surélévation du plan d'eau dans la zone de déferlement. Les gradients de contraintes de radiations vont être à l'origine de la surélévation du plan d'eau dans la zone de déferlement et des courants de dérive littorale.

Le module ST de la suite MIKE 21 est mis en œuvre pour la modélisation du transport sédimentaire dans l'anse de Morgat. MIKE 21 ST calcule le transport sédimentaire sur le fond et en suspension sous l'action combinée des courants et de la houle. Dans la zone de déferlement, les courants de retour sont inclus. Le modèle est déterministe et demande un minimum de calage. Les taux initiaux de sédimentation/érosion sont calculés à l'aide de l'équation de conservation de la masse sédimentaire. Un sable de diamètre médian $0.2 \mathrm{~mm}$, de gradation 1.4 et de porosité 0.4 est considéré sur l'ensemble du modèle.

Le calage du modèle hydrosédimentaire est réalisé en deux temps. Dans un premier temps, le modèle numérique est calé pour reproduire l'évolution historique de l'ensemble des plages de Morgat et du Portzic suite à la construction de la grande digue du large en 1971/72; les levés topo-bathymétriques de 1970 et de 1996/97 fournissent une évidence franche et marquée de cette évolution. Le modèle est ainsi calé pour cette période de sondage de 1970 à 1996/97. L'évolution morphologique très accentuée durant cette période présente un avantage considérable pour le paramétrage du modèle de transport sédimentaire contrairement à la période antérieure présentant un transport nul. Dans un deuxième temps, le calage du modèle numérique est validé sur la période avant construction des ouvrages; la stabilité de la plage de 1970 doit ainsi être reproduite avec le paramétrage et les conditions de forçage issus de la phase de calage du modèle.

\section{Résultats de la modélisation courantologique}

\subsection{Effet de la digue sur la houle et les courants}

La digue du large crée une zone abritée de la houle sur l'extrémité Sud Ouest des plages au fond de l'anse, avec une réduction progressive de l'agitation de l'Est vers l'Ouest. Suite à un gradient des contraintes de radiation du à la présence de la digue du large, une variation du niveau d'eau est observée près du rivage entre la plage de Morgat et du Portzic (Figure 3). Ce gradient est le plus accentué dans la zone de transition entre la partie abritée et exposée de l'ensemble des plages, située au centre de l'ensemble des plages. Le principal mécanisme responsable de l'érosion de la plage du Portzic est justement lié à cette variation du niveau d'eau 
le long du trait de côte : la surélévation du niveau d'eau face à la plage du Portzic provoque un basculement du plan d'eau qui, de suite, engendre un courant de houle orienté vers la plage de Morgat. Sous l'action de houle, ce courant alimente constamment la plage de Morgat avec les sédiments érodés de la plage du Portzic.
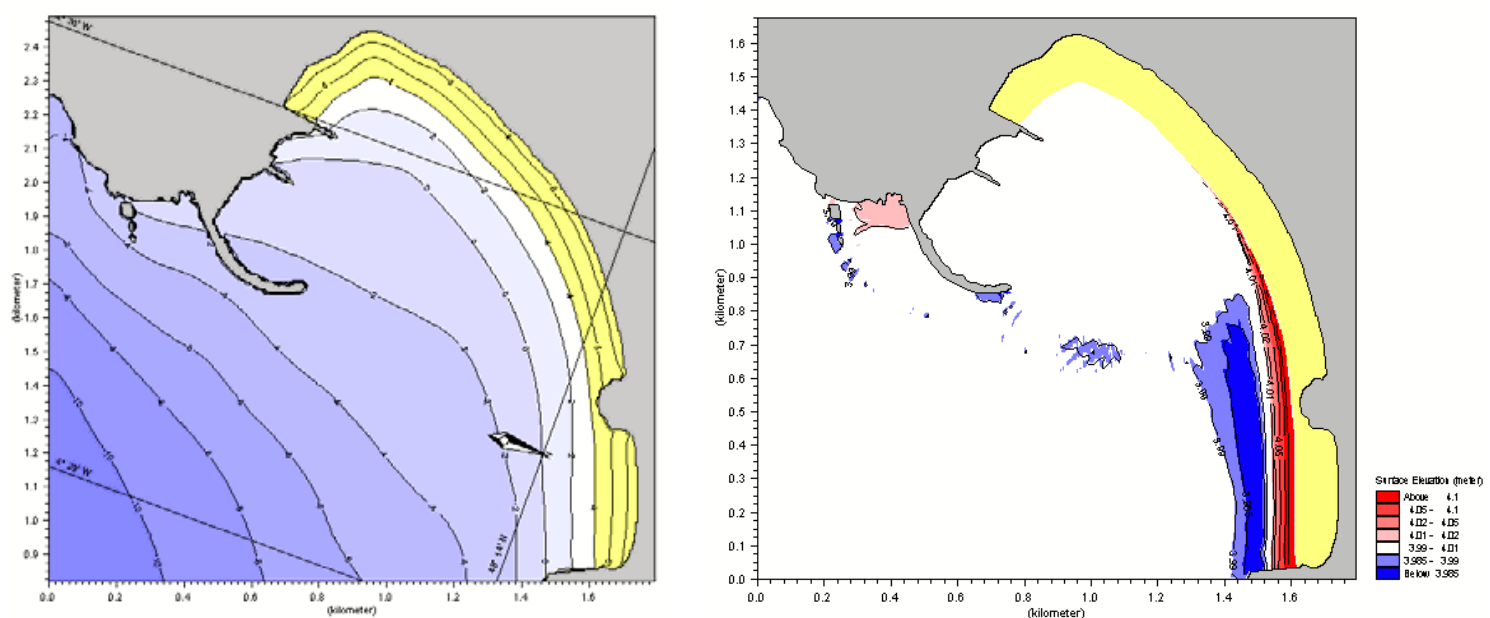

Figure 3: A gauche : Bathymétrie de 1970 (i.e. avant l'aménagement du port en 1971/72) avec digue du large. A droite : Variation de la surélévation du niveau d'eau pour un forçage aux frontières de $\mathrm{H} 1.69 \mathrm{~m}$ et d'incidence $190^{\circ}$ sur situation de mi-marée moyenne.

Une simulation de l'écoulement des courants avec et sans digue (voir Figure 4), illustre un changement considérable des courants face à la plage de Morgat : le basculement du plan d'eau présenté sur la Figure 3, engendre un courant de houle orienté vers le port de Morgat. Face à la plage du Portzic, l'effet de la digue sur l'écoulement des courants reste très limité. Il convient de noter l'aspect sens unique du transit littoral le long de la plage de Morgat. Suite à la construction de la digue du large les sédiments qui transitent vers le Sud-ouest devant la pointe de Rulianec sont définitivement piégés dans la plage de Morgat. Quelque soit la condition de forçage, le courant de houle est irréversiblement orienté vers cette plage.
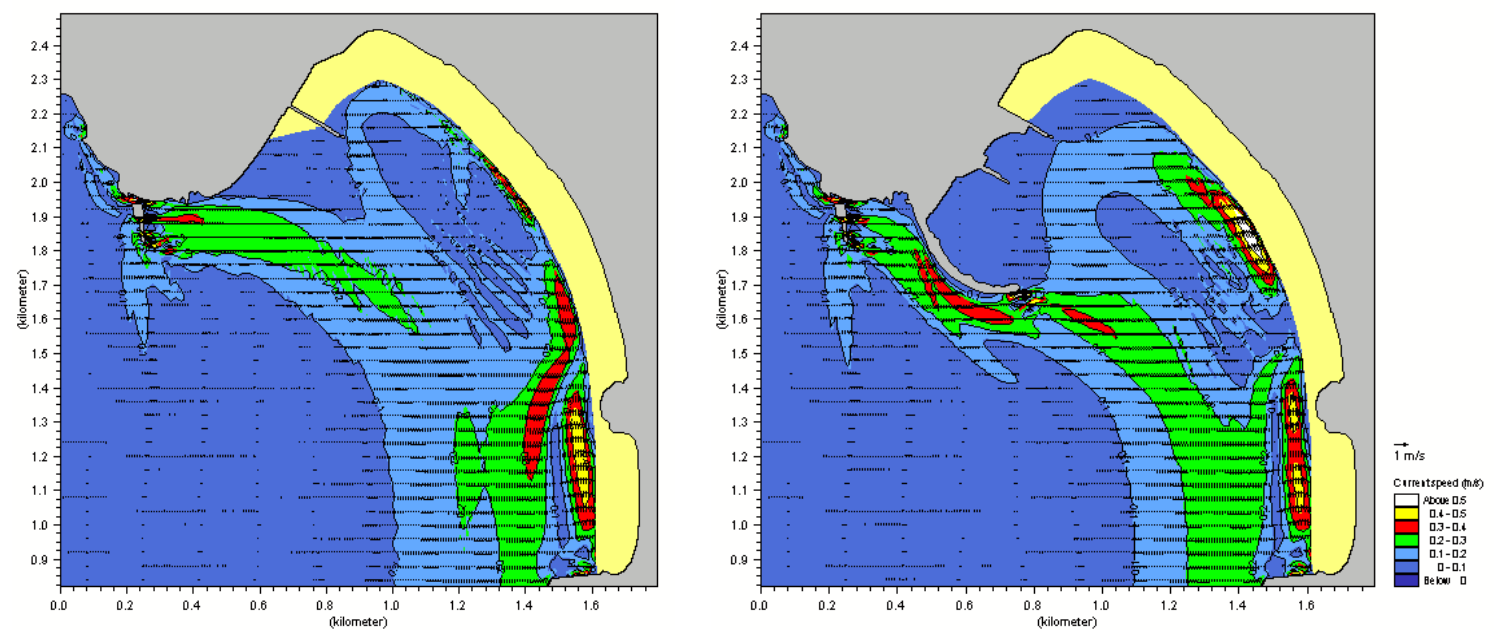

Figure 4: Evolution des courants pour un forçage aux frontières de $1.69 \mathrm{~m}$ et d'incidence $190^{\circ}$ sur situation de mi-marée moyenne; bathymétrie de 1970 sans (à gauche) et avec (à droite) digue du large. 


\subsection{Effet de la marée sur la houle et les courants}

Suite à la réfraction bathymétrique très sensible au niveau d'eau, l'incidence de la houle à la côte varie de plusieurs degrés en fonction de la marée. Cette variation engendre naturellement une variation de l'écoulement des courants de houle (Figure 5). L'effet de la marée est particulièrement important sur la plage du Portzic où la direction du courant de houle change de sens en fonction de la marée: pour un niveau d'eau de pleine mer (PM) d'une marée moyenne, le courant moyen est dirigé vers le Nord-est alors qu'à basse mer (BM), ce courant est dirigé vers le Sud-ouest. Sur la plage de Morgat, la marée a surtout un effet sur l'amplitude du courant de houle toujours en direction du port. Sur l'ensemble des plages, les courants de houle sont les plus forts pour un niveau d'eau de BM d'une marée moyenne.
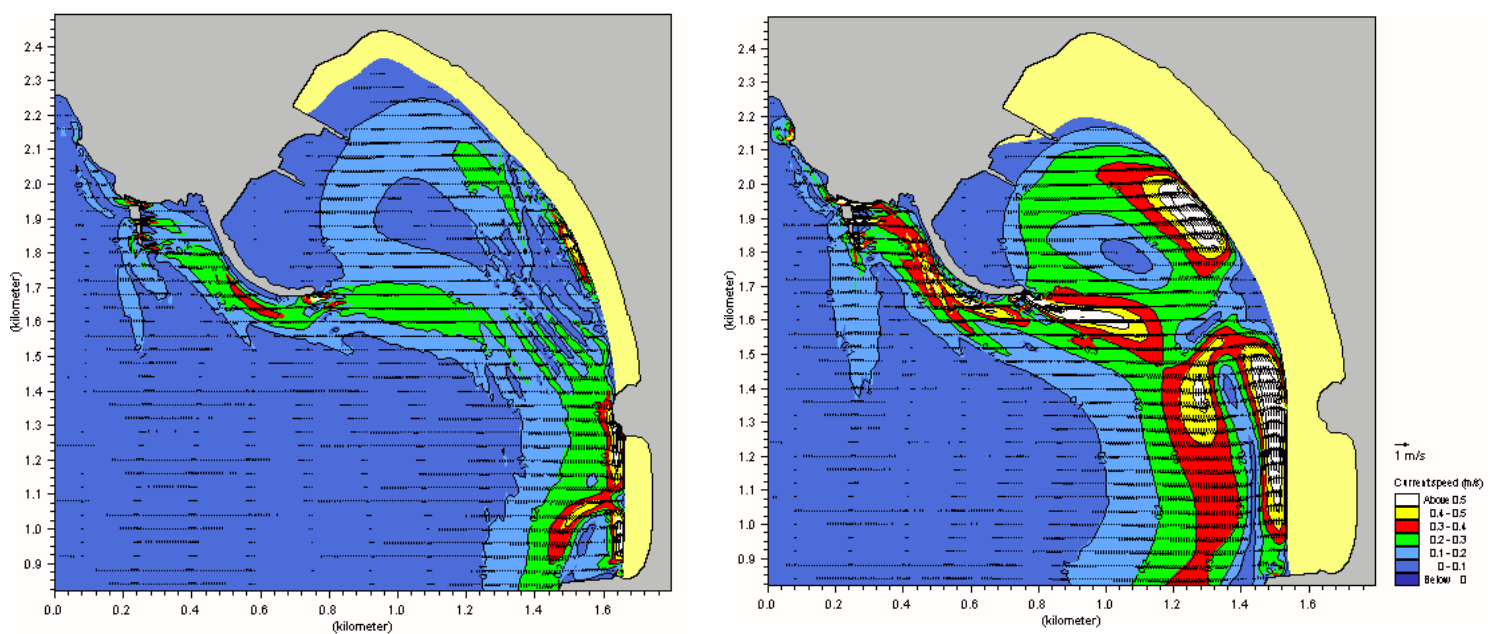

Figure 5: Evolution des courants pour un forçage aux frontières de $1.69 \mathrm{~m}$ et d'incidence $190^{\circ}$ sur situation de PM moyenne (à gauche) et de BM moyenne (à droite) ; bathymétrie de 1970 avec digue du large.

Ainsi, pour la compréhension du régime hydrosédimentaire de l'anse de Morgat, il est impératif de coupler dynamiquement la variation du niveau dans les simulations de propagation de houle et de courantologie. Ceci est mis en œuvre dans les simulations de transport sédimentaire présentées à suivre.

\section{Résultats de la modélisation du transport sédimentaire}

\subsection{Calage des conditions de forçage}

Le transit littoral, intégré du haut de l'estran jusqu'aux petits-fonds de $-2 \mathrm{~m}$ C.M., est moyenné spatialement sur une série de profils de plage perpendiculaires à la côte et temporairement sur un cycle de marée moyenne. Le transit à reproduire par calage du modèle est d'une part, de l'ordre de $15000 \mathrm{~m}^{3} /$ an [1] vers le port de Morgat (calculé sur un tronçon de plage au Sud-Ouest de la pointe de Rulianec) et d'autre part, un transit net nul sur la plage du Portzic (transit moyenné sur la longueur de cette plage).

Les premières séries de simulations bidimensionnelles du transport sédimentaire basées sur les conditions représentatives de forçage du Tableau 1 montre un léger transport sédimentaire vers la pointe des Grottes. A l'issue de nombreuses 
simulations de sensibilité, l'angle d'incidence permettant d'obtenir un transport résultant nul sur la plage de Portzic est $188.3^{\circ}$ et non pas $190^{\circ}$ comme estimé en première approche. Ainsi il s'avère que le pas de forçage de $15^{\circ}$ aux frontières choisi initialement pour les simulations de propagation de la houle ne permet pas la bonne représentation du transit littoral face à un forçage très étroit. A noter que la sensibilité de la plage du Portzic face à l'angle d'incidence de la houle n'est pas valable pour la plage de Morgat qui, suite à la construction de la grande digue, s'engraisse quelque soit l'angle d'incidence de la houle.

Sur la plage du Portzic, la capacité nette de transport résultant du forçage avec incidence de $188.3^{\circ}$ est nulle, or le transit littoral net à reproduire est de l'ordre de $15000 \mathrm{~m}^{3} / \mathrm{an}$. Ceci est lié au fait que le sable en provenance de la plage de Portzic est effectivement transporté vers la plage de Morgat alors qu'au niveau de la pointe de Rulianec, les courants de houle bloquent la possibilité aux sédiments de transiter en sens opposé. Pour obtenir ce double effet, 2 simulations par condition de houle ont été effectuées, chacune avec son angle d'incidence : $\mathbf{1 9 0}^{\circ}$ et $186.5^{\circ}$ à l'isobathe $-10 \mathrm{CM}$ - ces 2 angles sont définis de sorte le transport sédimentaire issu des 2 simulations (avec la moitié de la fréquence d'occurrence pour la condition) pour chacune des conditions justement ait un résultant nul sur la plage du Portzic. Pour chacun de ces deux angles d'incidence, une représentation graphique du transport sédimentaire moyenné sur un cycle de marée est illustrée dans la Figure 6.
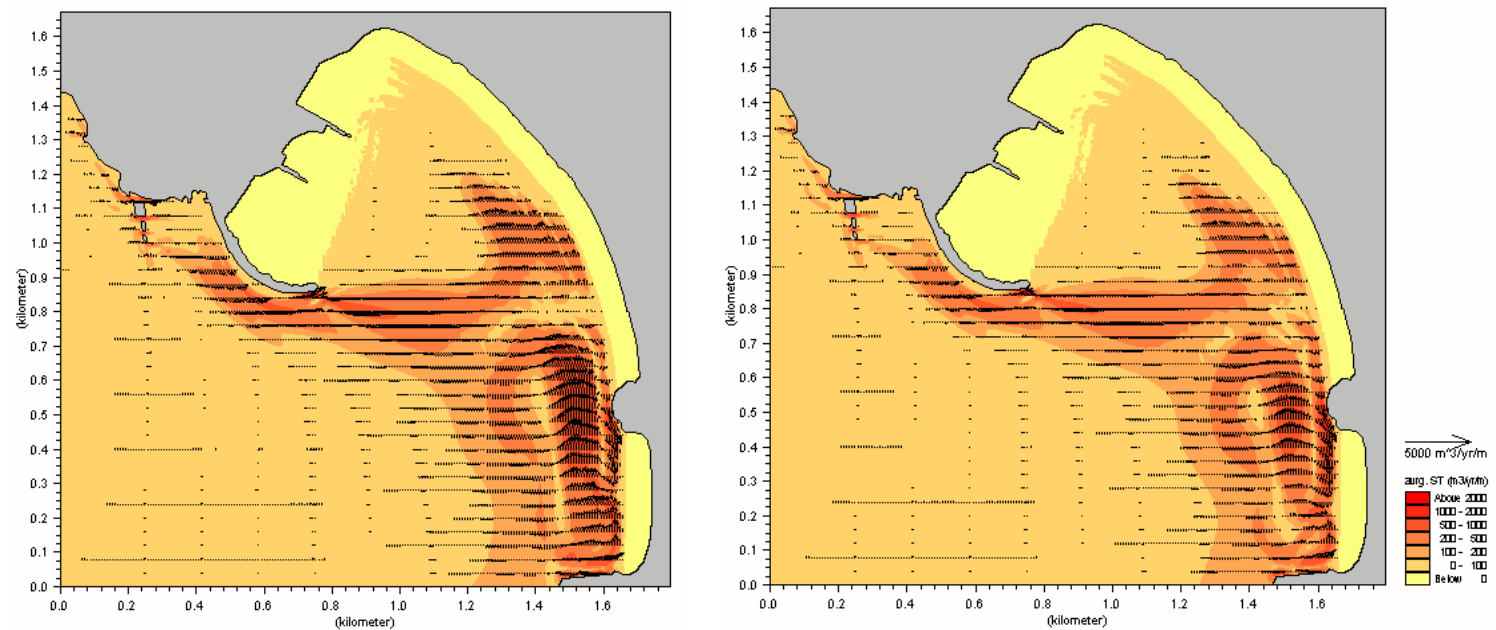

Figure 6: Transport sédimentaire moyenné sur un cycle de marée pour un forçage aux frontières de $1.69 \mathrm{~m}$ et d'incidence $190^{\circ}$ (à gauche) et $186.5^{\circ}$ (à droite) ; bathymétrie de 1970 avec digue.

Sur la plage du Portzic, l'effet distinct des 2 angles d'incidence par condition de houle est visible (Figure 6) : un angle de $190^{\circ}$ engendre un transit littoral brut vers la pointe des Grottes alors qu'un angle de $186.5^{\circ}$ engendre un transit brut vers la pointe de Rulianec. Sur la plage de Morgat, la direction du transit littoral reste inchangée pour ces 2 angles, mais la magnitude du transport varie légèrement.

\subsection{Stabilité de la plage de 1970}

Une validation du paramétrage et des conditions de forçage issus du calage du modèle est menée pour la période avant la construction des ouvrages, période 
caractérisée par la stabilité des plages du secteur étudié. La modélisation hydrosédimentaire doit ainsi pouvoir reproduire cette stabilité antérieure des plages.

Pour des tronçons de plage situés centralement dans chacune des plage de Morgat et du Portzic, le transit littoral, intégré du haut de l'estran jusqu'aux petits-fonds de $-2 \mathrm{~m} \mathrm{CM}$, est moyenné spatialement sur une série de profils de plage perpendiculaires à la côte et temporairement sur un cycle de marée moyenne : pour chaque plage, un transit net nul en résulte. Le transport sédimentaire résultant sous l'effet des deux hauteurs de houles moyenné sur un cycle de marée est présenté dans la Figure 7.
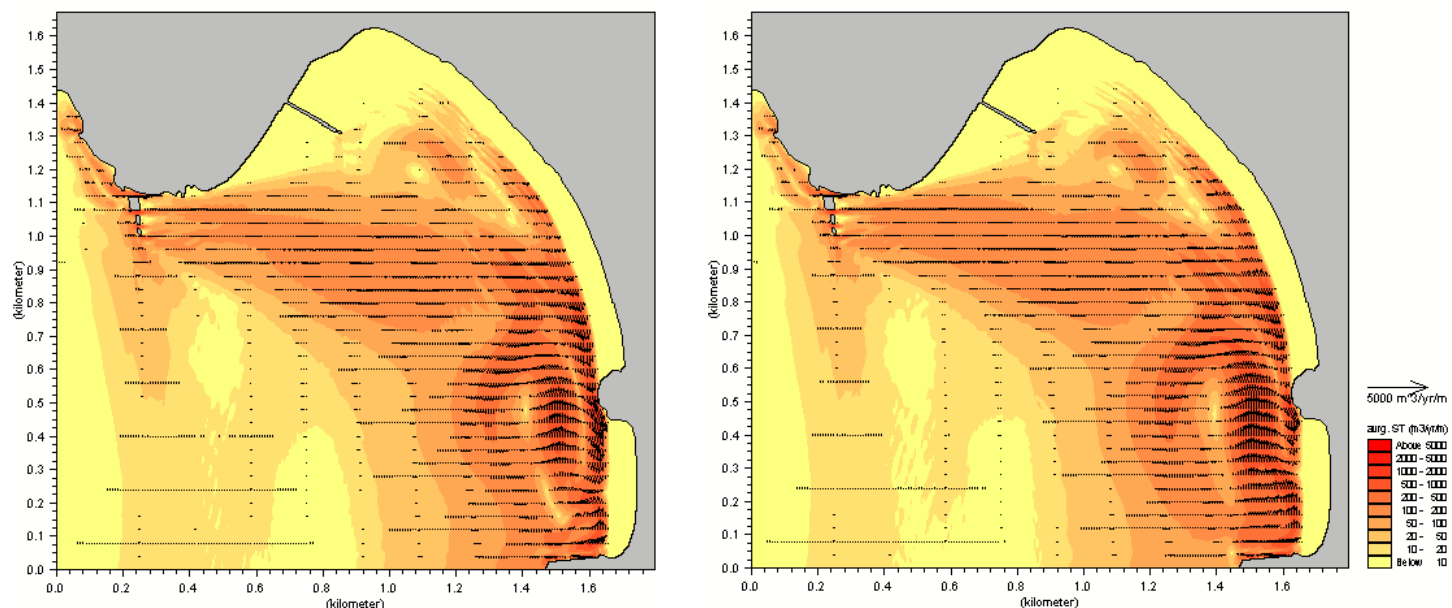

Figure 7: Transport sédimentaire résultant des 2 hauteurs de houle aux frontières (1.69 et 1.02 $\mathrm{m}$ ) moyenné sur un cycle de marée pour un forçage aux frontières d'incidence $190^{\circ}$ (à gauche) et $186.5^{\circ}$ (à droite) ; bathymétrie de 1970 sans digue.

Avec la disparition des courants de houles au niveau de la pointe de Rulianec engendrés par la digue du large, l'aspect sens unique du transit littoral n'existe plus : selon la direction d'incidence de la houle les sédiments peuvent transiter d'un sens à l'autre entre les deux plages.

L'effet de la marée sur le sens du transport sédimentaire reste globalement inchangé (i.e. avec et sans digue du large) sur la plage du Portzic : à marée haute, le transport est dirigé vers la pointe des Grottes et à marée basse, celui-ci est dirigé vers la pointe de Rulianec. La continuité du stock sableux sur cette plage est de suite assurée par un remaniement dans le profil. Sur la plage de Morgat, l'effet de la marée est moins important que la direction d'incidence de la houle : le transit littoral y est faible et globalement de magnitude opposée selon la direction d'incidence de la houle.

\section{Modélisation des aménagements futurs}

La conception de l'aménagement du site de Crozon-Morgat répond aux objectifs fondamentaux de la restructuration du site vis-à-vis de la réactivation de son port originel et de la restauration de sa plage. Elle résulte d'une concertation étroite menée avec le Maître d'Ouvrage et l'ensemble de ses partenaires. La solution repose sur un traitement de l'ensemble du secteur concerné (port et plages) 
contrant le déséquilibre de la dynamique sédimentaire qu'a entraînée la construction de la digue en enrochements dans le début des années 70 et une gestion appropriée de l'ensemble du stock sableux.

Les principaux éléments de cet aménagement sont :

(1) la réalisation d'une nouvelle jetée,

(2) le renforcement de la pointe de Rulianec

(3) le rechargement de la plage de Morgat et du Portzic.

La segmentation proposée de la plage est nécessaire pour assurer la stabilité de la plage $\mathrm{du}$ Portzic. Un paramètre impératif à l'établissement du nouvel équilibre sédimentaire de l'anse de Morgat est l'orientation de ces plages.

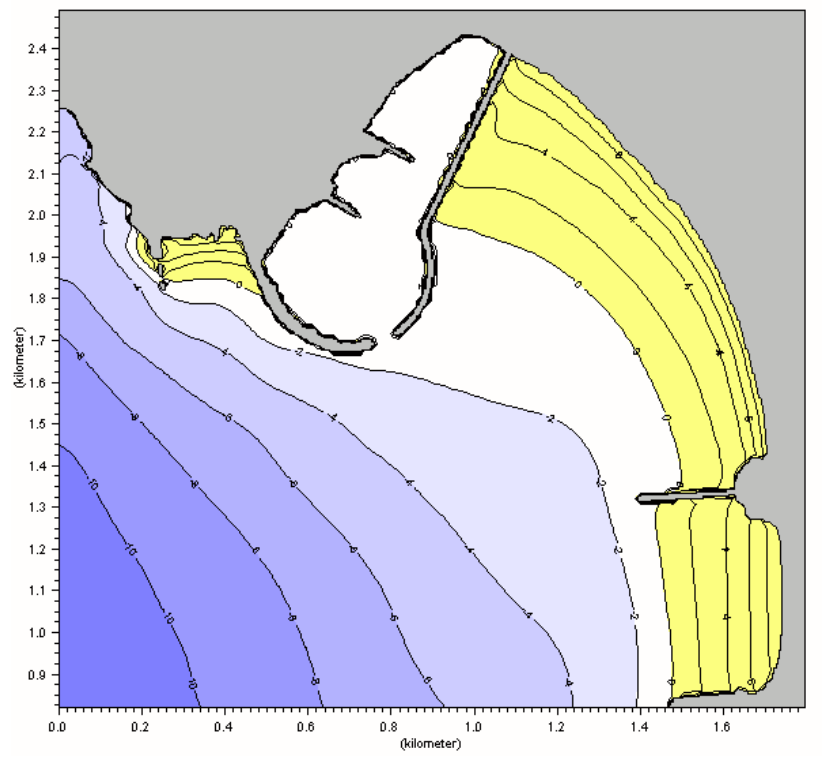

Figure 8: Configuration de l'aménagement proposé

De nombreuses simulations numériques à l'aide du modèle décrit auparavant ont ainsi été effectuées afin d'optimiser la variation de cette orientation le long du trait de côte.

\section{Conclusions}

La compréhension du fonctionnement hydrosédimentaire de la plage de Morgat est issue de la mise en auvre d'une modélisation numérique véritablement bidimensionnelle de la dynamique sédimentaire de l'anse de Morgat. L'évolution des plages du fond de l'anse a été étudiée en deux étapes. Une première étape a d'abord consisté à recréer dans le modèle numérique le transport solide observé de la plage du Portzic vers la plage de Morgat suite à la construction de la digue du port de plaisance (Phase de calage). Dans un second temps, le modèle a été mis en œuvre afin de s'assurer que les plages étaient globalement stables avant la construction de la digue (Phase de validation). Le modèle numérique ainsi mis en place permet alors de définir et valider un nouvel aménagement du site (avec notamment une extension du bassin portuaire) assurant la stabilité des plages de l'anse de Morgat.

\section{Références}

[1] Bernard Latteux, Plage et port de Morgat, 2003, Diagnostic sur l'état actuel, Rapport pour la commune de Crozon-Morgat, Décembre 2003.

[2] Service Hydrographique et Océanographique de la Marine (SHOM), 1994, Courants de marée de la côte Ouest de Bretagne - de Goulven à Penmarc'h. 